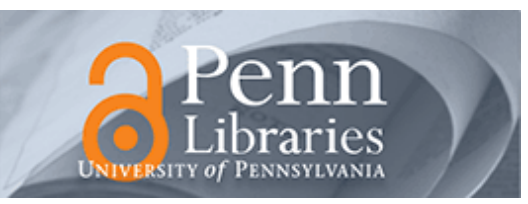

University of Pennsylvania

ScholarlyCommons

9-2009

\title{
Testing a Model of Patient Characteristics, Psychologic Status, and Cognitive Function as Predictors of Self-Care in Persons with Chronic Heart Failure
}

Janette Cameron

Linda Worrall-Carter

Barbara Riegel

University of Pennsylvania, briegel@nursing.upenn.edu

Sing Kai Lo

Simon Stewart

Follow this and additional works at: https://repository.upenn.edu/nrs

Part of the Behavioral Medicine Commons, Cardiology Commons, Cardiovascular Diseases

Commons, Circulatory and Respiratory Physiology Commons, Medical Humanities Commons, Neurology

Commons, Nursing Commons, and the Preventive Medicine Commons

\section{Recommended Citation}

Cameron, J., Worrall-Carter, L., Riegel, B., Kai Lo, S., \& Stewart, S. (2009). Testing a Model of Patient Characteristics, Psychologic Status, and Cognitive Function as Predictors of Self-Care in Persons with Chronic Heart Failure. Heart \& Lung, 38 (5), 410-418. http://dx.doi.org/10.1016/j.hrtlng.2008.11.004 


\title{
Testing a Model of Patient Characteristics, Psychologic Status, and Cognitive Function as Predictors of Self-Care in Persons with Chronic Heart Failure
}

\author{
Abstract \\ Objective \\ Self-care is a key component in the management of chronic heart failure (CHF). Yet there are many \\ barriers that interfere with a patient's ability to undertake self-care. The primary aim of the study was to \\ test a conceptual model of determinants of CHF self-care. Specifically, we hypothesized that cognitive \\ function and depressive symptoms would predict CHF self-care. \\ Methods \\ Fifty consecutive patients hospitalized with CHF were assessed for self-care (Self-Care of Heart Failure \\ Index), cognitive function (Mini Mental State Exam), and depressive symptoms (Cardiac Depression \\ Scale) during their index hospital admission. Other factors thought to influence self-care were tested in \\ the model: age, gender, social isolation, self-care confidence, and comorbid illnesses. Multiple regression \\ was used to test the model and to identify significant individual determinants of self-care maintenance \\ and management.
}

\section{Results}

The model of 7 variables explained $39 \%(F[7,42] 3.80 ; P=.003)$ of the variance in self-care maintenance and $38 \%(F[7,42] 3.73 ; P=.003)$ of the variance in self-care management. Only 2 variables contributed significantly to the variance in self-care maintenance: age $(P<.01)$ and moderate-to-severe comorbidity $(P<.05)$. Four variables contributed significantly to the variance in self-care management: gender $(P<$ $.05)$, moderate-to-severe comorbidity $(P<.05)$, depression $(P<.05)$, and self-care confidence $(P<.01)$. When cognitive function was removed from the models, the model explained less of the variance in selfcare maintenance $(35 \%)(\mathrm{F}[6,43] 3.91 ; P=.003)$ and management $(34 \%)(F[6,43] 3.71 ; P=.005)$.

Conclusion

Although cognitive function added to the model in predicting both self-care maintenance and management, it was not a significant predictor of CHF self-care compared with other modifiable and nonmodifiable factors. Depression explained only self-care management.

\section{Disciplines}

Behavioral Medicine | Cardiology | Cardiovascular Diseases | Circulatory and Respiratory Physiology | Medical Humanities | Medicine and Health Sciences | Neurology | Nursing | Preventive Medicine 
Testing a Model of Patient Characteristics, Psychological Status, and Cognitive Function as Predictors of Self-care in Persons with Chronic Heart Failure

Authors: JANETTE CAMERON, BN, CARDIOTHORACIC CERTIFICATE, MHLTHSCI (HEALTHED. \& PROMOTION) ${ }^{1}$, LINDA WORRALL-CARTER, RN, CORONARY CARE CERT, BeD., MeDC (PRELIM), PhD ${ }^{2}$, BARBARA RIEGEL, DNSc, RN, FAAN, FAHA ${ }^{3}$, SING KAI LO, BSC (HONS), PhD ${ }^{1}$, SIMON STEWART RN, BA, BN, GRAD DIP ED, PhD ${ }^{4}$.

${ }^{1}$ Faculty of Health, Medicine, Nursing \& Behavioural Sciences, Deakin University, Melbourne, ${ }^{2}$ School of Nursing \& Midwifery (Victoria), Faculty of Health Sciences, Australian Catholic University Limited, ${ }^{3}$ School of Nursing, University of Pennsylvania, Philadelphia, ${ }^{4}$ Preventative Cardiology, Baker IDI Heart and Diabetes Research Institute, Melbourne.

Acknowledgements:

Cameron, J., is supported by an NH\&MRC/NHF Public Health Post Graduate Scholarship With kind thanks to the nurse case managers across Eastern Health who supported this study.

Correspondence: Jan Cameron, St Vincent's Health / ACU National Centre for Nursing Research, PO Box 2900, Fitzroy Victoria, Australia 3065. Phone: + 61392883691

Fax:+61392883688 Email: S00072101@student.acu.edu.au 
Background: Self-care is a key component in the management of chronic heart failure (CHF). Yet there are many barriers that interfere with a patient's ability to undertake self-care.

Objective: The primary aim of the study was to test a conceptual model of determinants of CHF self-care. Specifically, we hypothesized that cognitive function and depressive symptoms would predict CHF self-care.

Methods: 50 consecutive patients hospitalised with CHF were assessed for self-care (SelfCare of Heart Failure Index), cognitive function (Mini Mental State Exam), and depressive symptoms (Cardiac Depression Scale) during their index hospital admission. Other factors thought to influence self-care were tested in the model: age, gender, social isolation, self-care confidence and comorbid illnesses. Multiple regression was used to test the model and to identify significant individual determinates of self-care maintenance and management.

Results: The model of seven variables explained $39 \%(F(7,42) 3.80 \mathrm{p}=0.003)$ of the variance in self-care maintenance and $38 \%(F(7,42) 3.73 \mathrm{p}=0.003)$ of the variance in self-care management. Only two variables contributed significantly to the variance in self-care maintenance: Age $(p<0.01)$ and moderate-to-severe comorbidity $(p<0.05)$. Four variables contributed significantly to the variance in self-care management: Gender $(\mathrm{p}<0.05)$, moderate-to-severe comorbidity $(p<0.05)$, depression $(p<0.05)$ and self-care confidence $(p<0.01)$. When cognitive function was removed from the models, the model explained less of the variance in self-care maintenance $(35 \%(F(6,43) 3.91 \mathrm{p}=0.003)$ and management $(34 \% \mathrm{~F}(6,43) 3.71 \mathrm{p}=0.005)$.

Conclusion: While cognitive function added to the model in predicting both self-care maintenance and management, it was not a significant predictor of CHF self-care compared to other modifiable and non-modifiable factors. Depression explained only self-care management.

Key words: cognitive impairment, self-care, depression, chronic heart failure 


\section{Background}

The prevalence of chronic heart failure (CHF) within the ageing populations in the Western World remains high ${ }^{[1,2]}$. The clinical trajectory of CHF is often characterized by chronic symptoms often interspersed with acute symptoms requiring hospitalisations for treatment ${ }^{[3]}$. It has been estimated that $50 \%$ of these re-hospitalisations are potentially preventable ${ }^{[4,5]}$ through better adherence with self-care practices ${ }^{[6,7]}$.

Given its importance in preventing potential fatal clinical crises, teaching patients self-care behaviours is a key non-pharmacological component in the management of CHF ${ }^{[\mathbf{8}]}$. Patients are taught self care strategies that enable them to engage in healthy behaviours such as following a low sodium diet and taking appropriate actions for symptoms ${ }^{[9]}$. This approach to patient education has been shown to substantially reduce hospitalisations ${ }^{[10]}$.

Despite a strong focus on patient education, however, the process of self-care is not readily learnt or understood by most patients ${ }^{[11]}$. Self-care decisions are often based upon the interaction between the person, the problem and their environment ${ }^{[12]}$ and factors known to enable self-care include patient characteristics, environmental factors and social support ${ }^{[13]}$. Barriers that can interfere with effective self-care and achievement of therapeutic goals include physical factors (increasing age ${ }^{[14,15]}$, co-morbidity ${ }^{[7,16]}$, and gender ${ }^{[14,17]}$ ) and psycho-social factors (social support ${ }^{[18]}$, depressive symptoms ${ }^{[13,19]}$, and self-efficacy $\left.{ }^{[20,21]}\right)$. Furthermore, the complexity and collection of symptoms associated with CHF cause functional and cognitive impairments that contribute to the challenge of living with this chronic illness ${ }^{[22-24]}$. Indeed, patients with $\mathrm{CHF}$ appear to have a 1.6-fold increase in risk for developing cognitive impairment than normal controls ${ }^{[25]}$. Patients with impaired cognition have poor health outcomes, even when enrolled in a CHF 
disease management program $(\mathrm{CHF}-\mathrm{MP}){ }^{[\mathbf{2 6}}$. One suggested explanation for this is that cognitive impairment decreases patients' abilities to assimilate self-care instruction and implement appropriate actions ${ }^{[27]}$ putting them at greater risk of re-hospitalisation. Despite acknowledging the impact of cognitive impairment on the decision-making process there remains a lack of evidence as to the specific reasons why most CHF patients fail to master self-care.

\section{Objectives}

The primary aim of this study was to test a conceptual model of factors drawn from the literature as potential determinants of CHF self-care (see figure 1). As cognitive impairment occurs frequently in patients with $\mathrm{CHF}$, we hypothesized that impaired cognitive function would be associated with poor CHF self-care practices. Another important factor hypothesized to be associated with poor CHF self-care was depressive symptoms. These and other potentially important factors identified from the literature were examined as potential determinants of CHF self-care in an elderly cohort of patients with the syndrome.

\section{Methods}

A convenience sample of consecutive patients hospitalised with CHF had an assessment of cognitive function, depressive symptoms, and self-care behaviours through administration of questionnaires during their index hospital admission. It was calculated that to detect a moderate effect size $\left(\mathrm{R}^{2}=0.3\right)$, with a significance level of 0.05 and allowing for seven predictor variables, a sample size of 50 would achieve 0.9 power. The study was approved by both the Health Network Research Committee and the University Human Research Ethics Committee and conforms to the principles outlined in the Declaration of Helsinki. 


\section{Sample}

Between June and December 2005, patients referred to the Chronic Heart Failure Management Program (CHF-MP) across one health network in Victoria, Australia were screened for study eligibility. As most patients are referred to the program during their in-patient episode, assessment of self-care was investigated at this time point so that recommendations could be made for applying and tailoring components of the CHF-MP to individual need.

The inclusion criteria for the study were a diagnosis of CHF using the clinical criteria from the Framingham study ${ }^{[28]}$ and moderate systolic dysfunction recorded on the echocardiogram report. As CHF affects mainly elderly persons, patients under 45 years of age were not included so that the results could be applicable to the wider CHF population. Patients were excluded from the study on the following basis: Neurological problems documented in the medical history (cerebral vascular accident, transitional ischemic attack, short-term memory loss or dementia); residing in a residential nursing home; and inability to answer questionnaires independently due to language barriers. Patients who met the inclusion criteria were approached by a hospital CHF case manager to participate in the study and then referred to the researcher.

Of the 96 patients with CHF screened, 7 patients (4\%) were excluded because English was not their primary language, 24 (14\%) were excluded because they had a history of a cerebral vascular disease or a transient ischemic attack (TIA), eight (5\%) were excluded for dementia, and 7 (4\%) declined to participate in the study. These 46 patients could not be compared to the 50 who were included because no further data were gathered on patients if they were excluded or had refused to participate. Fifty (52\%) patients met the inclusion criteria and agreed to participate in the study. 


\section{Procedure}

The primary investigator obtained informed consent from each patient. Overall, patient interviews were conducted 6 days $(\mathrm{SD} \pm 5)$ after hospital admission, usually while patients were still hospitalized. Instruments used to measure CHF self-care, cognitive function and depressive symptoms were administered at the time of the interview. Collected from the medical history were physical and social factors thought to influence self-care factors (age, gender, socially living with support or alone and comorbid illnesses).

\section{Instruments}

The Self-Care of Failure Heart Index (SCHFI) ${ }^{[29]}$ was developed to measure adherence to recommended CHF self-care behaviours, patient's decision making ability, and self-care confidence. The tool provides scores reflecting self-care maintenance (behaviours such as adhering to a low sodium diet), self-care management (the ability to recognise symptom changes, implement remedies and evaluate their effectiveness), and confidence with self-care (confidence to engage in each phase of the self-care process). According to Riegel \& Dickson ${ }^{[30]}$, maintenance and management reflect self-care, and confidence scores should be used to explain why some patients master self-care and others do not. Responses from each of the three self-care scales are transformed to 100 points each; higher scores reflect superior self-care. Scale scores $\geq 70$ are considered adequate self-care scores ${ }^{[31]}$. Psychometric testing of the SCHFI in 760 patients with CHF demonstrated adequate internal consistency of the self-care scales: self-care management (Cronbach's $\alpha .70$ ); and self-care self-confidence (Cronbach's $\alpha .82$ ). Low reliability for the selfcare maintenance scale (Cronbach's $\alpha .=.55$ ) was found by the instrument authors, which was explained by knowing that the behaviours reflected in the scale are largely independent ${ }^{[29]}$. 
The Cardiac Depression Scale (CDS) ${ }^{[32]}$, developed in Australia, is a psychometrically sound disease specific measure of depressed mood within cardiac populations. The instrument is made up of 26 items with 7 subscales (sleep, anhedonia, uncertainty, mood, cognition, hopelessness, inactivity). A seven-point response scale is used for each item, with positive items reverse scored. In instrument testing in a sample of CHF patients, $37 \%$ scored $>100$, a cut-off thought to indicate severe depressive symptoms. Psychometric analysis of the scale as well as correlation with other general depression measures has demonstrated its internal consistency and its validity $^{[32,33]}$. Testing in a sample of 141 cardiac patients ${ }^{[34]}$ demonstrated that a cut-off score $\geq 84$ had the same specificity as Beck's Depression Inventory cut-score of $\geq 9$ but had statistically superior sensitivity ( $97 \%$ vs $84 \% \mathrm{p}=0.004)$. The $\geq 84$ cut-off score was used in our study.

The Mini Mental State Examination (MMSE), ${ }^{[35]}$ one of the most widely used screening measures for dementia, was developed to estimate the severity of cognitive impairment. The MMSE consists of 30 questions that screen for orientation, short-term memory, concentration and visual spatial skills. The MMSE has satisfactory reliability, internal consistency and test-retest reliability, as well as high levels of sensitivity for moderate-to-severe cognitive impairment ${ }^{[36]}$. Although cut-points are usually reported at 23/24 there is also evidence that using higher-cut scores of 26/27, indicating less severe cognitive impairment, is of prognostic importance ${ }^{[26,37]}$. When long-term outcomes from a CHF-MP were compared against usual care in patients with less severe cognitive impairment, defined as a score of 19-26 on the MMSE, McLennan et al ${ }^{[26]}$ found that patients with reduced cognition were more likely to be re-admitted and less likely to survive the five year follow-up than those with MMSE scores $>26$ at the time of screening. This evidence supported our decision to use a cut-off score 26/27 to the current study. A significant advantage of 
this tool is that it can readily be used in the clinical setting by nurses or allied health professionals, taking only five to ten minutes to complete ${ }^{[38]}$.

The severity of co-morbid conditions was assessed using the Charlson Co-morbidity Index, which classifies co-morbidity based on the number and seriousness of co-morbid diseases. The weighted index of co-morbidity has proven to be a significant predictor of 1 -year survival with higher scores indicating greater risk of death ${ }^{[39]}$. Most diseases are assigned an index of 1 but more severe conditions are given a weighted score of 2, 3 or 6 . Overall index scores can be categorized as mild, moderate or severe co-morbidity.

\section{Analysis}

Analysis began with the calculation of descriptive statistics; normally distributed continuous data are presented as the mean \pm standard deviation. Percentages are presented with $95 \%$ confidence intervals (CI) where appropriate. In this sample, all patients reported symptoms of breathlessness or oedema in the previous month, allowing for computation of scores of all three components of the SCHFI: self-care management, self-care maintenance and self-care confidence.

Multiple regression analysis was used to assess the model of hypothesized factors and to determine the degree of variance in self-care maintenance and management scores that was explained by cognitive function, depression, co-morbidity (collapsed into two categories: low $=$ weighted index score 1-3 and moderate-to-severe $=$ weighted index scores $\geq 4$ ), gender, social situation and age. In the regression models, self-care confidence was used as a covariate rather than an outcome based on Riegel and Dickson's theory that confidence influences the ability to perform self-care ${ }^{[30]}$. The independent variables were entered using an enter method. SPSS (version 12.0.1 for Windows, Chicago, IL) was used for all analyses. 


\section{Results}

The socio-demographic and clinical characteristics of the group are presented in Table 1. Despite being elderly (mean age 73 years) most patients (60\%) were still married and only $28 \%$ lived alone. Females represented only $24 \%$ of the cohort. Half the group had been diagnosed with CHF more than two months previously and half were functionally compromised (NYHA class IIIIV). Twenty-seven (53\%) reported depressive symptoms with scores on CDS $\geq 84$. Eighteen (36\%) patients had a significant Charlson co-morbidity Index score $\geq 4$. Using the cut-point (26/27) on the MMSE, 18 patients (36\%) had evidence of potentially important cognitive impairment.

\section{Level of CHF Self-care}

The mean self-care scores for the group were: $67.8 \pm 17.3$ (range 25-100) for self-care maintenance; $50.04 \pm 16.64$ (range 16.68-91.74) for self-care management; and $62.00 \pm 19.98$ (range 25-100) for self-care confidence. In this sample, adequacy in self-care (defined as scores $\geq 70 \%$ on each component of SCHFI) was evident in only $52 \%$ of the sample in the performance of self-care maintenance. Only $12 \%$ were adequate in self-care management and $36 \%$ in self-care confidence.

Two multiple regression models were generated to determine the relationship between hypothesized predictors (age, gender, co-morbidity, cognitive function, depression, social situation and self-confidence) and the criterion variables: self-care maintenance and management scores. When self-care maintenance was regressed on the seven variables, they explained $39 \%(F(7,42)$ $3.80 \mathrm{p}=0.003$ ) of the variance in self-care maintenance scores (see Table 2). Older age made the largest contribution to self-care maintenance scores $(\beta=0.51, \mathrm{p}=0.001)$. To investigate the impact of cognitive function on self-care maintenance a second regression model was analysed removing MMSE score as a factor. The six variables explained 4\% less of the variance in self-care 
maintenance $(35 \%(F(6,43) 3.91, p=0.003)$ suggesting that cognitive function added to the model in predicting self-care maintenance.

When self-care management was regressed on the seven independent variables they explained $38 \%(F(7,42) 3.73 \mathrm{p}=0.003)$ of the variance in self-care management scores (see Table 3). Higher self-care confidence made the largest contribution to self-care management scores $(\beta=0.39, p<0.01)$. For every point increase in self-care confidence, there was a 0.39 point increase in self-care management. Three other variables contributed significantly to the variance: Male gender $(p<0.05)$, moderate-to-severe comorbidity $(p<0.05)$, depressive symptoms $(p<0.05)$. When MMSE score was removed in the second regression model the six variables explained $4 \%$ less of the variance in self-care management $(34 \%(F(6,43) 3.71 \mathrm{p}=0.005)$ suggesting that cognitive function added to the model in predicting self-care management.

\section{Discussion}

In the current study the hypothesized model of seven variables explained a significant amount of the variance in self-care maintenance and management. Cognitive function, the variable of particular interest, was not a statistically significant predictor but when this variable was removed from the model, less of the variance in self-care was explained by the remaining variables. Unique factors explained self-care maintenance and management. Older age and moderate-tosevere co-morbidity were significant determinants of self-care maintenance. In contrast male gender, moderate-to-severe co-morbidity, presence of depressive symptoms, and greater self-care confidence were significant predictors of self-care management. These models illustrate the influence of both modifiable and non-modifiable factors on self-care. Screening for these factors may help identify patients at greater risk of poor health outcomes so that follow-up strategies can be directed to those at greater risk of hospitalisations. 
The strongest predictor of self-care was a moderate-to-severe co-morbidity index, which was associated with higher self-care scores for both management and maintenance behaviours. These results are consistent with those of others ${ }^{[7,16]}$ who found that patients with more co-morbid illness and poorer functional class were better at practising self-care. Two possible explanations for this are that people with multiple co-morbid illnesses have had time to adapt to living with a chronic illness and are already conversant with self-care practices. Another possible explanation is that increasing symptoms and reduced functional capacity may motivate patients to be diligent with self-care. Arguably the increasing number of co-morbidities associated with aging can compete with patient's self-care management resources making the process of self-care overwhelming [40]. However, in this small sample we found better self-care in patients with more co-morbid illness and older age.

The finding that older age predicted self-care maintenance is similar to that of other researchers studying chronic diseases ${ }^{[41]}$ and CHF ${ }^{[14,15]}$. Qualitative study researchers ${ }^{[42]}$ have found similar results and have challenged the stereotype that elderly people are not interested in undertaking health promoting activities suggesting instead, that older patients are often keen to be actively involved in health promoting activities and self-care behaviours because they prefer to be independent and self-reliant. This perspective may help to explain some of our findings.

The correlation between gender and self-care management was surprising, albeit consistent with the results of other investigators. Chriss et al ${ }^{[14]}$ found that increased age and male gender were significant predictors of CHF self-care, although these variables explained little of the variance in self-care. Karlsson et al. ${ }^{[17]}$ also found significant differences in self-care between men and women using a researcher developed questionnaire to assess knowledge about CHF management. However, their nurse-based intervention increased knowledge scores in the women 
over a six month period, so that over time, gender differences were not statistically different (13.5 \pm 3.0 vs $12.2 \pm 3.7, \mathrm{p}=\mathrm{ns}$ ). This suggests that women may have more to gain from CHF-MP interventions that focus on teaching self-care management than men.

Our results also lend support that depression is associated with poor self-care, which may be linked to the interplay between self-efficacy and depression ${ }^{[20]}$. Tsay and Chao ${ }^{[21]}$ studied the effects of self-efficacy on depression in patients with CHF and found that self-efficacy was an important factor in patients' motivation to maintain physical function and an indirect influence on their depression. The model of Self-care in Chronic Illness ${ }^{[13]}$ also recognizes the important influence that psychological status can have on general self-care behaviors to promote health and prevent illness. Using this model in a qualitative study investigating factors that influenced selfcare, Schnell ${ }^{[19]}$ found that participants who described themselves as being upbeat were able to cope more effectively and practice self-care proficiently. Furthermore depression can result in neurocognitive dysfunction with impairments in memory and executive functions-effects that may indirectly influence self-care ${ }^{[43]}$.

We hypothesized that cognitive function would have a meaningful influence on self-care. It was therefore surprising to find that impaired cognitive function contributed to the model but individually did not significantly correlate with self-care maintenance or management. These results are consistent with early results from Pressler and colleagues (Susan Pressler, personal communication, November, 2007) who has suggested that CHF patients with impaired cognition are still able to perform self-care maintenance. We had hypothesized that patients who were cognitively intact would perform superior self-care management on the premise that this behaviour requires the cognitive ability to learn, perceive, interpret and respond to symptom changes ${ }^{\text {[27] }}$. These are higher order cognitive functions that are associated with executive functioning or 
problem solving skills originating from the pre-frontal cortex of the brain ${ }^{[44,45]}$. These cognitive functions are needed to construct and execute effective plans of action ${ }^{[46,47]}$. Failure to support this hypothesis could be due to the manner in which cognition was measured. Higher order cognitive functions are not measured by the MMSE and this limitation may help to explain why cognitive impairment was not a strong predictor of $\mathrm{CHF}$ self-care. Future studies investigating the relationship between self-care and cognitive function should use tools that are more sensitive in detecting mild impairments in higher order cognitive functions rather than screening measures for dementia.

Overall, self-care was low in this sample which may reflect the newness of the diagnosis. Only half the patients followed self-care maintenance instructions such as daily weighing; most did not understand the significance of their symptoms. Despite being able to evaluate their symptoms, they were not proactive in implementing any self-care actions. Other researchers ${ }^{[6,48,49]}$ also have found that despite being given appropriate instructions, adherence with self-care maintenance behaviours is particularly poor, even following a hospitalization for heart failure. This suggests that patient education alone does not improve self-care practices. Our results lend further evidence to the growing body of knowledge surrounding the number of non-modifiable factors that influence self-care practices. These factors must be considered when developing appropriate follow-up strategies and education programs for patients with CHF.

There were a number of limitations in this study that require comment. Females were not well represented in our study, which may have been due to the screening criteria to include only those with systolic dysfunction on echocardiography. Women often have a different etiology for CHF than males as they suffer more from hypertension than ischemic heart disease resulting in more diastolic dysfunction ${ }^{[28]}$. Social support was not formally assessed; we merely asked whether 
or not individuals were living alone. This short-coming may explain why we did not find, as others have found ${ }^{[18,50]}$, a correlation between social support and self-care. We also were unable to assess for a potential sampling bias because we did not collect demographic data on patients who qualified but were excluded due to refusal or exclusionary criteria. Despite these shortcomings the results from our study add to the body of evidence that many factors influence on an individual's ability to become competent with CHF self-care.

\section{Implications for research and practice}

The results of this small study add to the body of evidence that patients with heart failure overall have poor self-care maintenance and management behaviours in spite of the recent attention to improving this outcome. Our model of variables explained a significant amount of the variance in both self-care maintenance and management. Although, mild cognitive impairment was not statistically significant in this small sample, inclusion of the variable added variance to the predictive model. Executive function is required for insight, will, abstraction and judgmentcharacteristics used to construct and execute effective plans of action ${ }^{[46,47]}$. Future studies investigating the correlation between self-care and cognitive function should be conducted in a larger sample and with more sensitive screening tools for identifying mild cognitive impairments affecting frontal and temporal lobes of the brain. Identifying patients who are least likely to engage in self-care may help in the selection of follow-up strategies required to prevent hospital readmissions and improve health outcomes. This would also allow for improved resource management, with specific groups of patients being invited to participate in CHF-MP's.

\section{Conclusions}

Patients with CHF are encouraged to become active participants in their care which requires them to be able to interpret the importance of symptom changes, implement appropriate remedies 
and evaluate their effectiveness. To compound the self-care decision making process, patients with CHF are elderly, suffer concomitant co-morbid illnesses and often have changes in cognition, which may influence their capacity to become effective self-care managers. The results of this study illustrate factors associated with low levels of CHF self care in an Australian population. Clinical and demographic variables explained moderately large proportions of the variance in selfcare maintenance and management scores. Further research is recommended to investigate the interplay between non-modifiable factors and the application of CHF management strategies selectively directed to those in greatest need for improvements in self-care such as those who are depressed, cognitively impaired, young, female, and those with few co morbidities and less confidence in their ability to perform self-care. 


\section{References}

[1] Bleumink GS, Knetsch AM, Sturkenboom MCJM, Straus SMJM, Hofman A, Deckers JW, et al. Quantifying the heart failure epidemic: prevalence, incidence rate, lifetime risk and prognosis of heart failure The Rotterdam Study. European Heart Journal. 2004;25(18):1614-9.

[2] Rosamond W, Flegal K, Friday G, Furie K, Go A, Greenlund K, et al. Heart disease and stroke statistics--2007 update: a report from the American Heart Association Statistics Committee and Stroke Statistics Subcommittee. Circulation. 2007;115(5):e69-171.

[3] Krumholz HM, Chen YT, Wang Y, Vaccarino V, Radford MJ, Horwitz RI. Predictors of readmission among elderly survivors of admission with heart failure. Am Heart J. 2000;139(Pt 1):72-7.

[4] Cline C, Israelsson B, Willenheimer R, Broms K, Erhardt L. Cost effective management programme for heart failure reduces hospitalisation. Heart. 1998 Nov;80(5):442-6.

[5] Braunstein JB, Anderson GF, Gerstenblith G, Weller W, Niefeld M, Herbert R, et al. Noncardiac comorbidity increases preventable hospitalizations and mortality among Medicare beneficiaries with chronic heart failure. Journal of the American College of Cardiology. $2003 ; 42(7): 1226-33$.

[6] Moser DK, Doering LV, Chung ML. Vulnerabilities of patients recovering from an exacerbation of chronic heart failure. Am Heart J. 2005;150(5):984.

[7] Artinian N, Magnan M, Sloan M, Lange M. Self-care behaviors among patients with heart failure. Heart \& Lung : the Journal of Acute and Critical Care. 2002 May-Jun;31(3):161-72.

[8] Rich M. Management of heart failure in the elderly. Heart Fail Rev. 2002;7(1):89-97. 
[9] Riegel B, Carlson B. Facilitators and barriers to heart failure self-care. Patient Education and Counseling. 2002 Apr;46(4):287-95.

[10] McAlister FA, Stewart S, Ferrua S, McMurray JJ. Multidisciplinary strategies for the management of heart failure patients at high risk for admission: a systematic review of randomized trials. Journal of the American College of Cardiology. 2004 Aug 18;44(4):810-9.

[11] Horowitz CR, Rein SB, Leventhal H. A story of maladies, misconceptions and mishaps: effective management of heart failure. Social science \& medicine. 2004 Feb;58(3):631-43.

[12] Riegel B, Dickson VV. A situation-specific theory of heart failure self-care. Journal of Cardiovascular Nursing. 2008 05;23(3):190-6.

[13] Connelly CE. An empirical study of a Model of Self-Care in Chronic Illness. Clinical Nurse Specialist. 1993 Sep;7(5):247-53.

[14] Chriss PM, Sheposh J, Carlson B, Riegel B. Predictors of successful heart failure self-care maintenance in the first three months after hospitalization. Heart \& Lung: the Journal of Acute and Critical Care. 2004;33(6):345-53.

[15] Michalsen A, Konig G, Thimme W. Preventable causative factors leading to hospital admission with decompensated heart failure. Heart. 1998;80(5):437-41.

[16] Riegel B, Vaughan Dickson V, Goldberg LR, Deatrick JA. Factors associated with the development of expertise in heart failure self-care. Nursing Research. 2007;56(4):235-43.

[17] Karlsson M, Edner M, Henriksson P, Mejhert M, Persson H, Grut M, et al. A nurse-based management program in heart failure patients affects females and persons with cognitive dysfunction most. Patient Education \& Counseling. 2005;58(2):146-53. 
[18] Sayers SL, Riegel B, Pawlowski S, Coyne JC, Samaha FF. Social support and self-care of patients with heart failure. Annals of Behavioral Medicine. 2008;35(1):70-9.

[19] Schnell KN, Naimark BJ, McClement SE. Influential factors for self-care in ambulatory care heart failure patients: a qualitative perspective. Canadian Journal Of Cardiovascular Nursing = Journal Canadien En Soins Infirmiers Cardio-Vasculaires. 2006;16(1):13-9.

[20] Jerant A, Kravitz R, Moore-Hill M, Franks P. Depressive symptoms moderated the effect of chronic illness self-management training on self-efficacy. Medical Care. 2008 05;46(5):523-31.

[21] Tsay S, Chao Y. Effects of perceived self-efficacy and functional status on depression in patients with chronic heart failure. Journal of Nursing Research. 2002 12;10(4):271-8.

[22] Wolfe R, Worrall-Carter L, Foister K, Keks N, Howe V. Assessment of cognitive function in heart failure patients. European Journal of Cardiovascular Nursing. 2006;5(2):158-64.

[23] Stromberg A. The crucial role of patient education in heart failure. European Journal of Heart Failure. 2005;7:363-9.

[24] Bennett S, Sauve MJ, Shaw RM. A conceptual model of cognitive deficits in chronic heart failure. Journal of nursing scholarship : an official publication of Sigma Theta Tau International Honor Society of Nursing. 2005;37(3):222-8.

[25] Vogels RLC, Scheltens P, Schroeder-Tanka JM, Weinstein HC. Cognitive impairment in heart failure: A systematic review of the literature. European Journal of Heart Failure. $2007 ; 9(5): 440-9$. 
[26] McLennan S, Pearson S, Cameron J, Stewart S. Prognostic importance of cognitive impairment in chronic heart failure patients: Does specialist management make a difference? European Journal of Heart Failure. 2006 Feb;8(5):494-501.

[27] Dickson VV, Tkacs N, Riegel B. Cognitive influences on self-care decision making in persons with heart failure. Am Heart J. 2007;154(3):424-31.

[28] Ho K, Anderson K, Kannel W, Grossman W, Levy D. Survival After the onset of Congestive Heart Failure in Framingham Heart Study Subjects. Circulation. 1993 July;88(1):10715.

[29] Riegel B, Carlson B, Moser D, Seburn M, Hicks F, Roland V. Psychometric Testing of The Self-Care of Heart Failure index. Journal of Cardiac Failure. 2004;10(4):350-60.

[30] Riegel B, Dickson V. A situation-Specific Theory of Heart Failure Self-Care. Journal of Cardiovascular Nursing. 2008 May-June; 23(3):190-6.

[31] Riegel B, Dickson VV, Goldberg LR, Deatrick JA. Factors associated with the development of expertise in health failure self-care. Nursing Research. 2007;56(4):235-43.

[32] Hare D, Davis C. Cardiac Depression Scale: Validation of a new depression scale for cardiac patients. Journal of psychosomatic research. 1996;40(4):379-86.

[33] Birks Y, Roebuck A, Thompson D. A validation study of the Cardiac Depression Scale (CDS) in a UK population. British Journal of Health Psychology. 2004;9:15-24.

[34] Shi W, Stewart A, Hare D. Both major and minor depression can be accurately assessed using the Cardiac Depression Scale Heart Lung and Circulation. 2008 7-10 August;17(Supplement 3):S207. 
[35] Folstein M, Folstein S, McHugh P. "Mini-mental State". A practical method for grading the cognitive state of patients for the clinician. Journal of Psychiatric Research. 1975;12:189-98.

[36] Tombaugh T, McIntyre N. The Mini-Mental State Examination: A Comprehensive Review. Journal of American Geriatric Society. 1992;40(9):922-35.

[37] Gussekloo J, Westendorp RG, Remarque EJ, Lagaay AM, Heeren TJ, Knook DL. Impact of mild cognitive impairment on survival in very elderly people: cohort study. BMJ : British medical journal. 1997 Oct 25;315(7115):1053-4.

[38] Lezak M, Howieson DB, Loring DW. Neuropsychological Assessment. 4th ed. New York: Oxford University Press 2004.

[39] Charlson ME, Pompei P, Ales KL, MacKenzie CR. A new method of classifying prognostic comorbidity in longitudinal studies: development and validation. Journal of Chronic Diseases. 1987;40(5):373-83.

[40] Kerr EA, Heisler M, Krein SL, Kabeto M, Langa KM, Weir D, et al. Beyond comorbidity counts: how do comorbidity type and severity influence diabetes patients' treatment priorities and self-management? Journal Of General Internal Medicine: Official Journal Of The Society For Research And Education In Primary Care Internal Medicine. 2007;22(12):1635-40.

[41] Schreurs KMG, Colland VT, Kuijer RG, de Ridder DTD, van Elderen T. Development, content, and process evaluation of a short self-management intervention in patients with chronic diseases requiring self-care behaviours. Patient Education and Counseling. 2003;51(2):133-41.

[42] Berg G, Sarvimaki A, Hedelin B. Hospitalized older peoples' views of health and health promotion. International Journal of Older People Nursing. 2006 Mar;1(1):25-33. 
[43] Butters MA, Whyte EM, Nebes RD, Begley AE, Dew MA, Mulsant BH, et al. The nature and determinants of neuropsychological functioning in late-life depression. Arch Gen Psychiatry. 2004 Jun 61(6):587-95.

[44] Xu G, Meyer JS, Thornby J, Chowdhury M, Quach M. Screening for mild cognitive impairment (MCI) utilizing combined mini-mental-cognitive capacity examinations for identifying dementia prodromes. Int J Geriatr Psychiatry. 2002;17(11):1027-33.

[45] Naugle RI, Kawczak K. Limitations of the Mini-Mental State Examination. Cleveland Clinic Journal of Medicine. 1989;56(3):277-81.

[46] Royall DR, Lauterbach EC, Cummings JL, Reeve A, Rummans TA, Kaufer DI, et al. Executive control function: a review of its promise and challenges for clinical research. A report from the Committee on Research of the American Neuropsychiatric Association. The Journal Of Neuropsychiatry And Clinical Neurosciences. 2002 Fall;14(4):377-405.

[47] Fellows LK. The cognitive neuroscience of human decision making: a review and conceptual framework. Behavioral \& Cognitive Neuroscience Reviews. 2004 Sep;3(3):159-72.

[48] Evangelista LS, Doering LV, Dracup K, Westlake C, Hamilton M, Fonarow GC. Compliance behaviors of elderly patients with advanced heart failure. Journal of Cardiovascular Nursing. 2003;18(3):197-208.

[49] Ni H, Nauman D, Burgess D, Wise K, Crispell K, Hershberger R. Factors influencing knowledge of and adherence to self-care among patients with heart failure. Archives of Internal Medicine. 1999 Jul 26;159(14):1613-9. 
[50] DiMatteo MR. Social support and patient adherence to medical treatment: a meta-analysis. Health Psychology. 2004 03;23(2):207-18. 
Table 1. Socio-demographic and clinical characteristics of cohort

\begin{tabular}{|l|c|}
\hline Socio demographic characteristics & $\mathrm{n}=50$ \\
\hline Age (years) & $73( \pm 11)$ \\
\hline Women (\%) & $12(24 \%)$ \\
\hline Married (\%) & $30(60 \%)$ \\
\hline Social situation - Lived alone (\%) & $14(28 \%)$ \\
\hline Education level <10 years (\%) & $15(30 \%)$ \\
\hline $\begin{array}{l}\text { When interview conducted (mean days from hospital } \\
\text { admission) }\end{array}$ & $6( \pm 5)$ \\
\hline Clinical characteristics & $25(50 \%)$ \\
\hline New Diagnosis of CHF (\%) & $32(64 \%)$ \\
\hline Mild co-morbidity, (\%) & $18(36 \%)$ \\
\hline Cognitive Impairment, MMSE <27 (\%) & $25(50 \%)$ \\
\hline NYHA classification moderate-to-severe, (\%) & $8(16 \%)$ \\
\hline Hypotension, BP <100mmhg (\%) & $27(53 \%)$ \\
\hline Renal impairment, serum creatinine $>110 u m o l / 1(\%)$ & $(38 \%)$ \\
\hline Aneamic, serum Hb <10 g/l (\%) & \\
\hline Depression (any level) (\%) & $24 \%)$ \\
\hline
\end{tabular}


Table 2. Multiple Regression analysis predicting Self-care Maintenance $(n=50)$

\begin{tabular}{lccccc} 
& $\begin{array}{c}\text { Standardized } \\
\text { Coefficients } \\
\text { Beta }\end{array}$ & Partial R & F Value & P & R Square \\
\hline Predictor variables & 0.51 & 0.49 & & $<0.01$ & \\
\hline Mge & -0.01 & -0.02 & & 0.92 & \\
Male gender & 0.34 & 0.34 & 3.80 & 0.02 & 0.39 \\
Significant co- & 0.23 & 0.23 & & 0.13 & \\
morbidity & -0.16 & -0.17 & & 0.28 & \\
$\begin{array}{l}\text { Cognitive function } \\
\text { Depression }\end{array}$ & 0.15 & 0.18 & & 0.25 & \\
$\begin{array}{l}\text { Social situation - living } \\
\text { with support }\end{array}$ & 0.07 & 0.07 & & 0.64 & \\
$\begin{array}{l}\text { Self-care self- } \\
\text { confidence }\end{array}$ & & & &
\end{tabular}


Table 3. Multiple Regression analysis predicting Self-care Management $(n=50)$

\begin{tabular}{lccccc} 
& $\begin{array}{c}\text { Standardized } \\
\text { Coefficients }\end{array}$ & & & & \\
Predictor variables & Beta & Partial R & $\begin{array}{c}\mathrm{F} \\
\text { value }\end{array}$ & $\mathrm{P}$ & $\begin{array}{c}\mathrm{R} \\
\text { Square }\end{array}$ \\
\hline \hline Age & 0.02 & 0.02 & & 0.91 & \\
Male gender & -0.33 & -0.36 & & 0.02 & \\
Significant co-morbidity & 0.33 & 0.33 & & 0.03 & \\
Cognitive function & 0.25 & 0.25 & & 0.09 & \\
Depression & 0.32 & 0.34 & 3.73 & 0.04 & 0.38 \\
Social situation - living & & & & & \\
with support & -0.006 & -0.007 & & 0.97 & \\
Self-care confidence & 0.39 & 0.39 & & $<0.01$ &
\end{tabular}


Figure 1: Conceptual model of factors that may influence CHF self-care

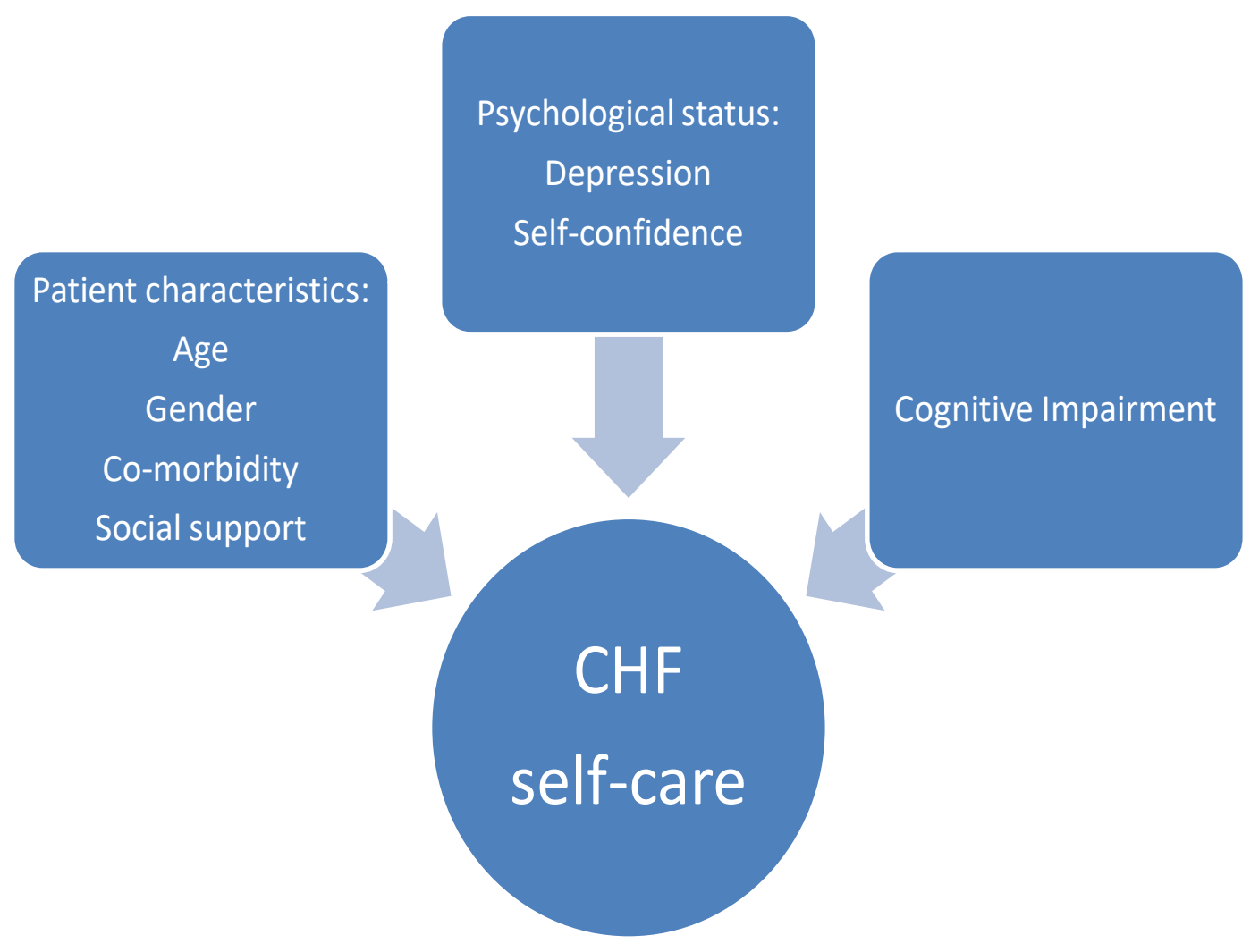

